\title{
Single Pulley Double-strand Anchor Suture Fixation of the Coronoid Process in Terrible Triad of the Elbow Injury
}

\author{
Yih-Wen Tarng ( $\square$ qm1047@ms35.hinet.net) \\ Department of Orthopaedics, Kaohsiung Veterans General Hospital, Kaohsiung city, Taiwan \\ Kai-Cheng Lin \\ Kaohsiung Veterans General Hospital
}

\section{Technical advance}

Keywords: Terrible triad, Coronoid process avulsion fracture, Single pulley double-strand suture, Suture anchor, Pull out suture

Posted Date: January 29th, 2021

DOl: https://doi.org/10.21203/rs.3.rs-155381/v1

License: (c) (1) This work is licensed under a Creative Commons Attribution 4.0 International License.

Read Full License 


\section{Abstract}

Background: Terrible triad of the elbow injury is difficult to manage, and the role of the coronoid process in instability is very important. We describe a simple, modified suture technique to fix a coronoid process fragment using suture anchor fixation.

Methods: Eight patients ( 3 female and 5 male) with coronoid process injuries with the fragment involving $<50 \%$ of the total height (Reagan-Morrey type I/II) in terrible triad of elbow injury were included. Patients were treated operatively via a lateral Kocher's approach, and coronoid process fractures were repaired with a single pulley double-strand suture technique. Structures were addressed in a sequential fashionthe coronoid process, radial head, lateral ulnar collateral ligament.

Results: All patients were treated with the single pulley double-strand anchor suture technique and the coronoid process fragment was found to be in good contact with the original avulsion site using the method. The final Mayo Elbow Performance Score was excellent $(>90)$ in 6 patients and good (between 85 and 89$)$ in 2 patients.

Conclusions: The single pulley double-strand suture tie method using a suture anchor is a less invasive and simpler fixation method for the repair of coronoid process fractures in patients with terrible triad of the elbow injuries, and results in good outcomes.

Level of evidence: Level IV; Case Series; Treatment Study

\section{Background}

Terrible triad of the elbow is difficult to manage because it is a combination of a coronoid process avulsion fracture, radial head fracture, and posterolateral dislocation of the elbow, which results in extreme elbow instability. ${ }^{1-4}$ In many cases, surgical fixation is incomplete leading to instability, limited range of motion (ROM), and elbow stiffness. ${ }^{5}$ The avulsed coronoid process fragment is responsible for most loss of fixation because many surgeons consider it a Regan-Morrey type I/II coronoid process tip fracture. ${ }^{6}$ Conservative treatment is recommended for Morrey type $\mathrm{I} / \mathrm{II}$ coronoid process fractures, but they typically occur with simple posterior dislocation without any collateral ligament injury. ${ }^{6}$ In most cases the anterior capsule is intact, and closed reduction is sufficient because the elbow is stable. The trauma and injury in terrible triad, however, is different. In terrible triad of the elbow, there is anterior capsule disruption with coronoid process tip avulsion, and a lateral ulnar collateral ligament (LUCL) avulsion tear that results in posterolateral rotation instability. ${ }^{7,8}$ The relative instability of the elbow is due to loss of the anterior buttress of the elbow if fixation of coronoid process tip is not performed. $2,7,8$ Therefore, coronoid process fixation is not only bone fixation, but also serves as anterior capsule repair, and is very important for achieving elbow stability. ${ }^{8-10}$

Some orthopaedic surgeons only fix the radial head/neck fractures and repair the LUCL, and then use dynamic ESF to prevent anterior-posterior instability. Coronoid process fixation may not be performed 
because it is very difficult to fix a coronoid process fracture. Several special methods, however, have been described to fix coronoid process fractures including a posterior pull out suture, ${ }^{9-11}$ suture lasso fixation, ${ }^{4}$ lag screws, ${ }^{3,8,9}$ a precontoured locking plate, ${ }^{12,13}$ and suture anchors. ${ }^{14,15}$ Although these methods can be effective, they are very complicated and have a steep learning curve. In this report, we describe a simple, modified suture technique to fix a coronoid process fragment using suture anchor fixation. It allows the repair of the three components of the terrible triad of the elbow via a single lateral Kocher's approach.

\section{Methods}

With approval of the Institutional Review Board of Kaohsiung Veterans General Hospital, we conducted a retrospective study of patients with coronoid process avulsion fracture of terrible triad injuries of the elbow in our department between January 2013 and December 2017. We enrolled eight patients (5 males and 3 females) from 23 to 59 years of age. All were right-hand dominant, and injuries occurred in the left elbow in three patients and in the right elbow in five patients. The mechanisms of injury included a fall from a motorcycle, a fall down stairs, and a motor-vehicle collision. Initial radiographic imaging revealed posterolateral rotatory elbow dislocation with concomitant radial head and coronoid process fractures in all patients. All patients were initially treated in the emergency department with closed reduction and long-arm splinting immobilization. Patients underwent surgical intervention as soon as possible before soft tissue swelling progressed in order to prevent compartment syndrome. In three patients, however, surgery was delayed approximately 16-24 hours. The radial head fracture patterns were simple in seven patients, and open reduction and fixation with two $2.0 \mathrm{~mm}$ screws was performed in these patients. One patient with a comminuted radial head received a radial head prosthesis replacement. All coronoid process injuries included one or two fragment transverse fractures of the coronoid involving less than $50 \%$ of the total height (Reagan-Morrey type I/II). All patients were treated operatively with a lateral Kocher's approach to the elbow. The coronoid process fractures were repaired with a suture anchor inserted into the coronoid process base with the strands passing through the coronoid process fragment and anterior capsule. The single pulley double-strand suture technique were used for fixation (Fig. 1). Structures were generally addressed in a sequential fashion-first the coronoid process, then the radial head, and finally the LUCL repair.

\section{Surgical technique}

Surgical dissection was performed for adequate exposure of the anterior aspect of proximal ulna, including the coronoid fracture fragment and trochlea notch via the lateral Kocher's approach. In all cases, the lateral collateral ligament and common extensor muscle origin were completely avulsed from the lateral epicondyle of humerus, and facilitated into the anterior and medial joint before soft tissue swelling. In all cases, the anterior capsule was disrupted and the avulsed coronoid process fragment could be identified. The anterior impaction fracture was usually found over the radial head. Pushing the radial head posterior allowed improved visualization of the coronoid fracture. Subperiosteal dissection 
along the anterior distal humerus allowed further exposure of ulnohumeral joint, facilitating debridement of loose bodies. An attempt at reduction of the fragment with a dental pick was performed when necessary to assess the adequacy of bony stability, as well as to plan the placement of the suture anchor(s).

The drill guide was placed in the fracture bed at the appropriately oriented angle, and the pilot hole for the initial suture anchor is made. The suture anchor was then placed into the hole in the standard fashion, and fixation was tested to ensure no pull-out (Fig. 1, A). The non-absorbable suture tails were then passed through the anterior capsule, tensioned, and tied over the anterior capsule and fragment, thus achieving reduction (Fig. 1, B). The anchor was placed in this fashion along the breadth of the coronoid fracture base, with two strands incorporating the fracture fragment and anterior elbow capsule from medial to lateral. Each anchor contained two different color strands, and the same color suture was tied from deep to superficial using the traditional technique.

Our technique, the single pulley double-strands method, uses one double-loaded suture anchors (Twinfix Ultra Ti 5.5-mm suture anchor, non-absorbable No. 2 braided polyethylene; Smith \& Nephew Endoscopy, Andover, MA, USA) with two different color strands, as mentioned above. The different color strands pass through the fracture fragment and the anterior capsule from inferior to superior. First, a knot is tied in the two different color suture tails (Fig. 2, A), and then the tails of the other color sutures are pulled. The coronoid process fragment is reduced and compressed to its original location by the previous knot when the second set of suture tails is pulled (Fig. 2, B). The two threads slide via the suture anchor, providing a pulley effect. Another knot is then tied using the left hand to pull one strand and slide the knot while the avulsed fragment is compressed with the right hand (Fig. 3, A). Confirmation that the fragment is attached to the original avulsion site with the forearm in supination and the elbow at $45^{\circ}$ flexion (Fig. 3 , B). Passive flexion-extension motion of the elbow was then checked after fixation. Immediate improvement of elbow alignment and stability was noted after repair of the coronoid injury.

Additional procedures, such as open reduction and $2.0 \mathrm{~mm}$ screw fixation of the anterior fragment of radial head fracture (Fig. 4), or prosthesis replacement of a comminuted fracture of the radial head (Fig. 5) were performed when necessary. Finally, lateral collateral ligament and common extensor muscle origin repair were performed. The wound was irrigated and closed layer by layer. The wound was dressed, and a long arm splint was applied with the elbow in $90^{\circ}$ of flexion.

\section{Postoperative care and follow-up}

The rehabilitation protocol was as follows. Passive ROM with continuous passive motion (CPM) were begun on postoperative day 7 in a 60-90-120 fashion. Active ROM was begun when pain could be tolerated. After discharge from the hospital, patients were followed-up at 2 weeks, 6 weeks, and 3 and 6 months after surgery, and annually thereafter. Fracture reduction was determined by plain radiographs taken immediately after surgery, and radiographs were performed every 6 weeks until bone union was achieved. Functional results were evaluated by Mayo Elbow Performance Score (MEPS) and active ROM 
at the last outpatient clinic visit. The MEPS was divided into 4 parts (pain, arc of motion, stability, function) and scored as excellent (score > 90), good (score 75-89), fair (score 60-75), and poor (score< $60)$.

\section{Results}

Postoperative radiographs (elbow anteroposterior and lateral) showed good bone contact in all cases of single pulley double-strand fixation (Fig. 4, B). Compare with the previous cases using a traditional suture anchor fixation method, mild floating of the fracture fragments was noted (Fig. 4, A). The final MEPS score was excellent (>90) in six patients and good (between 85 and 89 ) in two patients. Active range of motion (AROM) in supination/pronation ranged from $67-86^{\circ}$ for supination and $77-90^{\circ}$ for pronation, and flexion/extension ranged from $112-135^{\circ}$ for flexion and $10-30^{\circ}$ for extension lag. Two patients had extension lag of $25-30^{\circ}$, but quality of life was not affected (by patient self-report). Finally, the arc of flexion- extension average ranged from $18-127^{\circ}, 83^{\circ}$ of pronation, and $80^{\circ}$ of supination, which constitute a functional range of elbow motion. All patients reported they were satisfied with their ability to perform the activities of daily life and return to previous work.

\section{Discussion}

Terrible triad of the elbow injury is difficult to manage because it is a triple injury, and it often lead to instability, contracture, re-operation, and progression to arthrosis of the elbow. ${ }^{16}$ The role of the coronoid process in instability after a terrible triad injury is very important. Most elbow instability is the result of loss of the anterior buttress if coronoid process fixation is not performed. ${ }^{17}$. Lack of full extension due to loss of the anterior buttress can lead to flexion contracture, stiffness, or re-dislocation of the elbow. Even if a coronoid process fracture without dislocation heals, incongruity of the joint is usually present resulting in progressive arthrosis of the elbow

We believe there are two reasons why many surgeons do not repair the coronoid process. First, most surgeons think fixation in Morrey type $\mathrm{I} / \mathrm{II}$ coronoid process avulsion fractures is not necessary. Second, it is difficult to fix a tiny avulsed fragment. Some surgeons use dynamic ESF to keep the elbow reduced during rehabilitation. But dynamic ESF is inconvenient for patients in their daily life. According to the Morrey principle, fixation of Morrey type I/II coronoid process fractures is not necessary, but in most cases the injury is a simple fracture- dislocation without any collateral ligament injuries. In a terrible triad injury, however, a Morrey type I/II coronoid process fracture is always combined with a LUCL or MCL injury, and extreme instability is present. For this reason, we believe all injuries that occur in the terrible triad of the elbow must be repaired to obtain stability of the elbow and good functional outcomes.

There is a general belief that it is very difficult to fix the coronoid process fragment. Techniques described for coronoid process fixation include a posterior pull-out suture, suture lasso fixation, lag screw fixation, use of a precontoured locking plate, and suture anchors. Lag screw and precontoured locking plates are suitable Morrey type III larger fragments. Although the pull-out suture and suture lasso fixation techniques 
are suitable for type I/II smaller fragments, usually two incisions or a global posterior incision are needed, and the techniques have a steep learning curve. The suture anchors technique is a novel idea, and suitable for type I/II smaller fragments. It is also suitable for terrible triad injury via the same lateral incision to fix the fracture fragment and anterior capsule. Unfortunately, it is often a dilemma as to have the elbow in flexion or extension when the knot is slid to compress the fragment in the traditional suture anchor technique. Manipulation is relatively easy when the elbow is extended, but it is difficult to attach the fragment to the original fracture site. When the elbow is extended and the knot is slide we have always found the fragment floating above the coronoid base on postoperative radiographs because of brachialis traction, although the elbow is stable. On the other hand, putting the elbow in flexion will counteract brachialis traction, but manipulation is more difficult and the fragment is still found floating on postoperative radiographs (Fig. 4, A). However, the method we have described for the suture anchors of type I/II coronoid process avulsion fractures in terrible triad injuries requires only one incision via a lateral Kocher's approach. If additional access to the coronoid is needed, anterolateral dissection may be performed by elevating the wrist and common digital extensors, as well as the supinator.

Our single pulley double-strand suture tie method is different from a traditional anchor suture tie. Two independent knots form two points to compress the avulsed fragment, and this resists the brachialis traction force that occurs with the traditional suture technique. Our technique uses one anchor with two pairs of knots that form a square to reduce and compress the avulsed fragment. Compression in the shape of a square is better than two points because it creates a broad area of bone contact. Of course, the "double pulley" technique ${ }^{18}$ is stronger than our method with respect to rotator cuff repair, but the coronoid process base is too small for 2 screws. Based on our experience with our technique, however, 1 screw is enough for fixation and to resist the pull of the brachialis. With our technique, radial head fracture reduction and fixation, or replacement, and LUCL repair can be performed after coronoid process fixation using the same incision. None of our patients required a medial side approach, or dynamic ESF assist after surgery.

In our experience, terrible triad patients should receive surgery as soon as possible because the procedures are more difficult once soft tissue swelling occurs. We prefer the injury to surgery time to be less than 8 hours. It is difficult to repair the coronoid process using the traditional suture tie technique with an anchor suture when there is soft tissue swelling due to a delay in surgery. In past cases, 2 incisions are typically used, a lateral and an anteromedial approach. However, our method can be performed with only one incision (lateral approach) even if there is soft tissue swelling.

In conclusion, the single pulley double-strand suture tie method using a suture anchor is a less invasive and simpler fixation method for the repair of coronoid process fractures in patients with terrible triad of the elbow injuries. We believe all terrible triad injury patients should have coronoid process fixation to achieve optimal outcomes.

\section{Abbreviations}


ROM

range of motion, LUCL:lateral ulnar collateral ligament, ESF:external skeletal fixator, CPM:continuous passive motion, MEPS:Mayo Elbow Performance Score, AROM:Active range of motion, MCL:medial collateral ligament.

\section{Declarations}

Ethics approval and consent to participate

With approval of the Institutional Review Board of Kaohsiung Veterans General Hospital, and this is a retrospective study and review medical record.

\section{Consent for publication}

Not applicable.

\section{Availability of data and materials}

The datasets used and analyzed during the current study are available from the corresponding author on reasonable request.

\section{Competing interests}

No authors of this study have any financial and personal relationships with other people or organizations, which could result in an inappropriate influence of this study.

\section{Funding}

Not applicable.

\section{Authors' contributions}

YWT and KCL participated in the design of the study. YWT conceived of the study, participated in its design and coordination, and drafted the manuscript. All authors read and approved the final manuscript.

\section{Acknowledgements}

Not applicable.

\section{Author details}

${ }^{1}$ Department of Orthopaedics, Kaohsiung Veterans General Hospital, Kaohsiung city, Taiwan

2 Department of Orthopaedics, National Defense Medical Center, Taipei city, Taiwan 


\section{References}

1. O'Driscoll SW, Jupiter JB, Cohen MS, Ring D, McKee MD. Difficult elbow fractures: pearls and pitfalls. Instr Course Lect. 2003;52:113-134.

2. O’Driscoll SW, Jupiter JB, King GJ, Hotchkiss RN, Morrey BF. The unstable elbow. Instr Course Lect. 2001;50:89-102.

3. Tashjian RZ, Katarincic JA. Complex elbow instability. J Am Acad Orthop Surg. 2006;14(5):278-286.

4. Garrigues GE, Wray WH 3rd, Lindenhovius AL, Ring DC, Ruch DS. Fixation of the Coronoid Process in Elbow Fracture-Dislocations. J Bone Joint Surg Am. 2011;93(20):1873-1881

5. Morrey BF. Complex instability of the elbow. Instr Course Lect.1998;47:157-164.

6. Doornberg JN, Ring D. Coronoid fracture patterns. J Hand Surg [Am]. 2006;31(1):45-52.

7. Ring D, Jupiter JB. Reconstruction of posttraumatic elbow instability. Clin Orthop Relat Res. 2000;370:44-56

8. Seijas R, Joshi N, Hernandez A, Catalán JM, Flores X, Nieto JM. Terrible triad of the elbow - role of the coronoid process: a case report. J Orthop Surg (Hong Kong). 2005;13(3):296-299.

9. Zeiders GJ, Patel MK. Management of unstable elbows following complex fracture-dislocations the "terrible triad" injury. J Bone Joint Surg Am. 2008;90 (Suppl 4):75-84

10. Pugh DM, Wild LM, Schemitsch EH, King GJ, McKee MD. Standard surgical protocol to treat elbow dislocations with radial head and coronoid fractures. J Bone Joint Surg Am. 2004;86(6): 1122-1130.

11. Forthman C, Henket M, Ring DC. Elbow dislocation with intra-articular fracture: the results of operative treatment without repair of the medial collateral ligament. J Hand Surg Am. 2007;32(8): 1200-1209.

12. Doornberg JN, Ring DC. Fracture of the anteromedial facet of the coronoid process. J Bone Joint Surg Am. 2006;88(10):2216-2224.

13. Doornberg JN, de Jong IM, Lindenhovius AL, Ring D. The anteromedial facet of the coronoid process of the ulna.J Shoulder Elbow Surg. 2007;16(5):667-670.

14. Pai V, Pai V. Use of suture anchors for coronoid fractures in the terrible triad of the elbow. J Orthop Surg (Hong Kong). 2009;17(1):31-35

15. Clarke SE, Lee SY, Raphael JR. Coronoid fixation using suture anchors. Hand (N.Y). 2009;4(2):156160.

16. McKee MD, Pugh DM, Wild LM, et al. Standard surgical protocol to treat elbow dislocations with radial head and coronoid fractures. Surgical technique. J Bone Joint Surg Am. 2005;87 (Suppl 1):2232.

17. Mathew PK, Athwal GS, King GJ. Terrible triad injury of the elbow: current concepts.J Am Acad Orthop Surg. 2009;17(3):137-151.

18. Murena L, Canton G, Falvo DA, Genovese EA, Surace MF, Cherubino P. The "Double-Pulley" Technique for Arthroscopic Fixation of Partial Articular-Side Bony Avulsion of the Supraspinatus Tendon: A Rare 
Figures
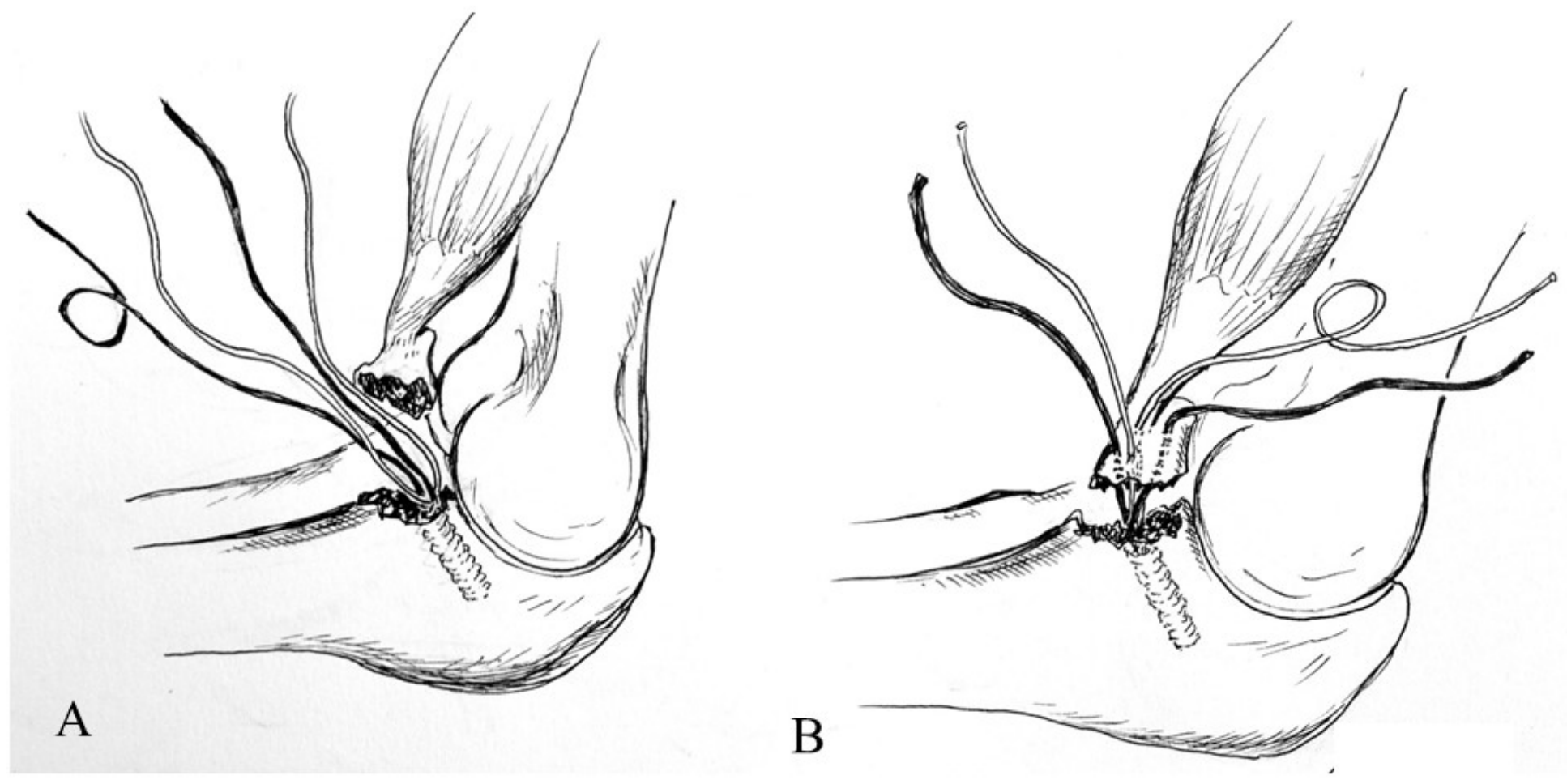

Figure 1

A) The suture anchor is placed in the standard fashion, and fixation is tested to ensure no pull-out. B) The non-absorbable strand tails were passed through the coronoid process fragment or anterior capsule from inferior to superior. 

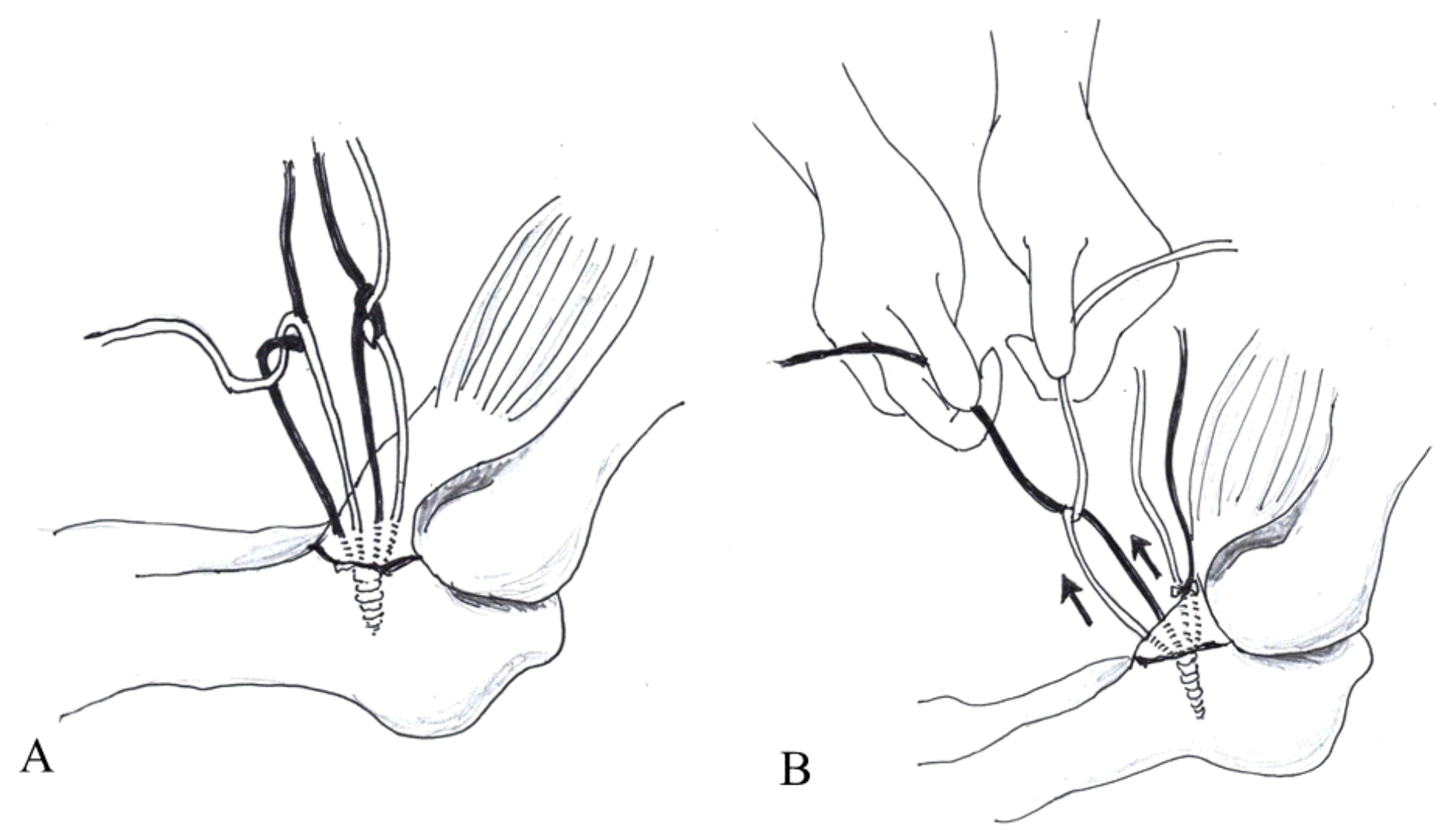

Figure 2

A) The different color threads pass through the fracture fragment and anterior capsule, are tensioned, and tied over the anterior capsule and fragment, achieving reduction. B) The first knot was tied from two different color strands, and another knot was then pulled to push the knot down to the fragment via the eyelets of the suture anchor. 


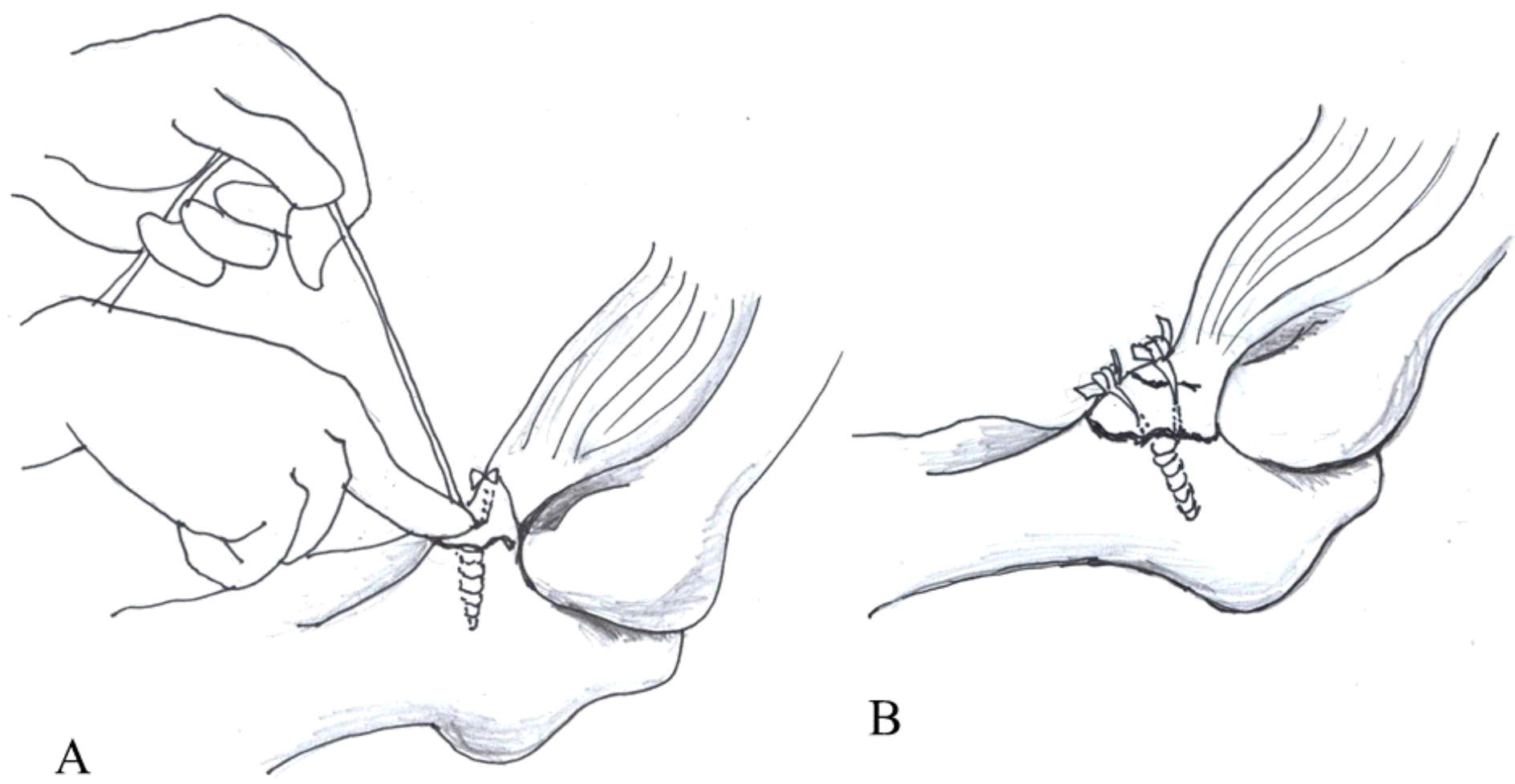

Figure 3

A) Another knot is tied using the left hand to pull one strand and slide the knot, and compression of the avulsed fragment is achieved by the right hand. B) The single pulley double-strands suture tie method uses one anchor with two pairs of knots. A square is formed to reduce and compress the avulsed fragment, rather than two independent knots. 

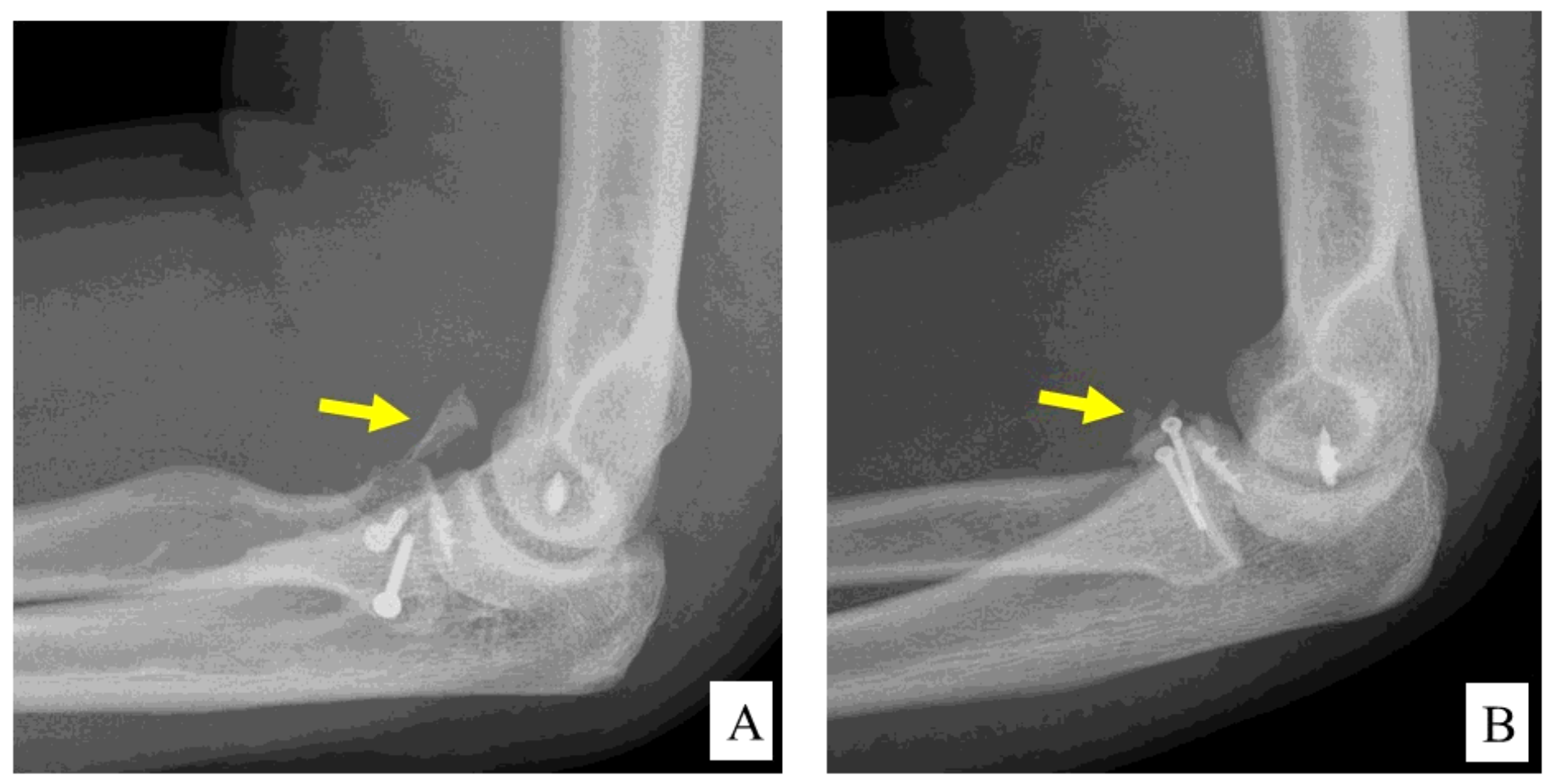

Figure 4

A) The coronoid process fragment (yellow arrow) was usually found to be floating using the traditional method of tying via one lateral incision because of delayed surgery and soft tissue swelling in past case. B) The coronoid process fragment (yellow arrow) was noted to be in good contact with the original avulsion site using the single pulley double-strand method despite delayed surgery and soft tissue swelling. 


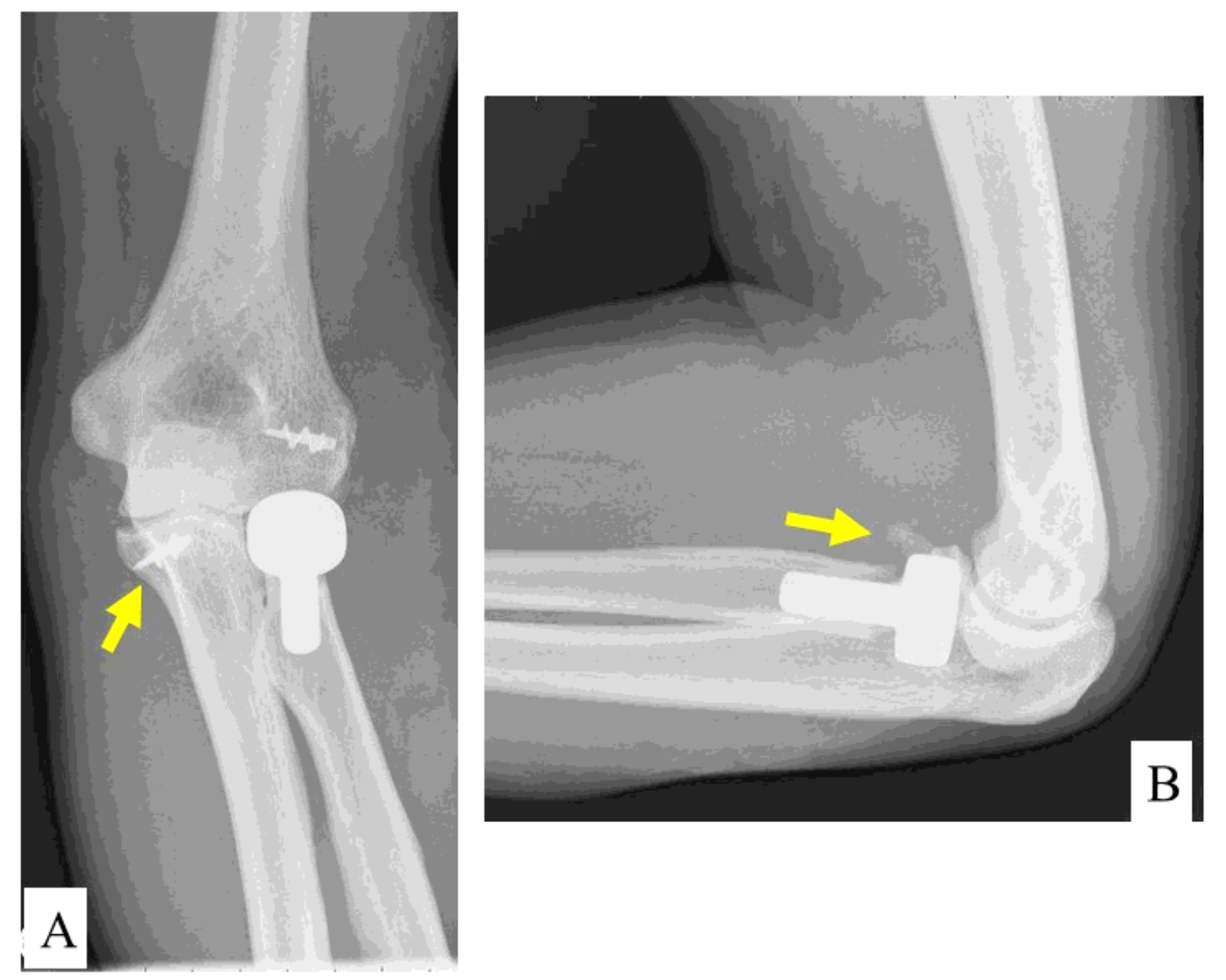

\section{Figure 5}

The facilitated into the anterior and medial joint after radial head resection due to comminuted fracture of radial head. The coronoid process fragment was easily fixed before radial head replacement. A) Suture anchor (yellow arrow) in anteroposterior view. B) The coronoid process fragment (yellow arrow) in good contact with the avulsion site in lateral view. 